\title{
Der Wassergehalt des fertigen Froscheies und der Mechanismus der Bildung seiner Hülle im Eileiter.
}

\author{
Von \\ G. Wetzel. \\ (Aus dem Königlichen anatomischen Institut zu Breslau.) \\ Eingegangen am 27. September 1908.
}

In meiner letzten Mitteilung über die stoffliche Zusammensetzung der Eier einiger Tiere habe ich daranf hingewiesen, daß es oft unmöglich ist, nur die Eimasse selbst zur Untersuchung zu bekommen. Zuweilen muß man die Eihüllen mit rerarbeiten oder ein andres Mal, wenn man die reifen Eier im Ovar nimmt, auch die uibrigen Bestandteile dieses Organs, also die zahlreichen jüngeren Eier nebst Follikelzellen und das Bindegewebe des Ovars, sowie die Follikelhüllen der reifen Eier selbst. Die Gallerthülle wurde z. B. von mir mit analysiert beim Seeigelei, der Eierstock bei der Seespinne.

Ähnliche Schwierigkeiten bietet auch das Froschei. Die fertigen Froscheier findet man, schon von der Gallerthülle umgeben, zur Laichzeit im untersten Abschnitte der beiden Eileiter vor. Sie lassen sich in diesem Zustande am besten in großen Mengen gewinnen. Die Hülle ist dünn, da sie wenig wasserhaltig ist. Nach der Eiablage quillt sie, wie bekannt, unter starker Wasseraufnahme zu mächtigen Dimensionen auf. Diese Massen sind zur Untersuchung schon infolge ihres Volumens, dann auch wegen der Verunreinigung durch zahlreiche pflanzliche Bestandteile, die der Gallertmasse anhaften, wenig. geeignet. Außerdem findet man beim Sammeln der Eimassen sehr verschiedene Entwicklungsstadien vor.

Das aus dem untersten Abschnitt des Eileiters gewonnene Material besteht also aus Eisubstanz und der Hülle. Da auch diese Wasser enthält, ist an solchen Eiern eine genaue Feststellung des Wassergehaltes nicht möglich. 
Die Art, wie die Eier aus dem Ovar in den Uterus übergehen, ermöglicht indessen beim Frosch die Erlangung fertiger Eier, die nur mit einer äußerst feinen Dotterhaut bekleidet sind. Wie Swammerdan zuerst beobachtet hat und neuerdings besonders von Nussbavm $\left.{ }^{1}\right)$ eingehend untersucht worden ist, gelangen die Eier aus den geplatzten Follikeln zunächst in die Bauchhöhle und von da in den Eileiter. Die Bauchhöhle ist an bestimmten Bezirken mit einem Flimmerepithel versehen, welches die wesentlichste Rolle bei der Beförderung der Eier in den Eileiter spielt. Einzelheiten des Vorganges sind bei Lebrux ${ }^{2}$, Nussbaum $u$. a. dargestellt. Im Eileiter werden die Eier dann mit der Gallerthülle umkleidet und sammeln sich in seinem untersten dünnwandigen Abschnitt an, der sich dabei sackartig erweitert.

Öffnet man ein Weibchen gerade zu der Zeit, wo die Eier sich auf der Überwanderung durch die Bauchhöhle befinden, so kann man eine große Anzahl fertiger Eier ohne Hüllen erlangen.

Die mir von meinem Fänger gelieferten Froschweibchen (Rana fusca) wiesen leider fast sämtlich die Eier schon mit Gallerthille versehen im Uterus auf. Nur in einem einzigen guinstigen Fall erhielt ich Bauchhöhleneier. Bei diesem Exemplare fand sich gleichzeitig noch ein abnormer Zustand an einem Eileiter vor.

Der unterste Abschnitt des linken Eileiters erwies sich beim Öffnen in der gewohnten Weise prall mit Eiern gefüllt. Im rechten fanden sich an dieser Stelle nur einige wenige Eier mit Gallerthülle, im übrigen eine große Anzahl tauber Eier, d. h. leerer Gallertkugeln. Eine größere Anzahl nackter Eier lag an versehiedenen Stellen der Bauchhöhle angehäuft.

Die Ursache für das abweichende Verhalten der rechten Seite dürfte in einer Verlegung des Zuganges zum Eileiter zu suchen sein. Näheres konnte ich hierïber nicht ausfindig machen. Die Verlegung war augenscheinlich von einer Verzögerung in der Abwanderung der Bauchhöhleneier in den schon überfüllten linken Eileiter begleitet.

An den Bauchhöhleneiern konnte ich nun in einwandfreier Weise den Wassergehalt des fertigen Froscheies feststellen. Ferner konnte ich Wasserbestimmungen an den mit Gallerthülle versehenen Eiern nnd an den leeren Eihüllen ausführen. Außerdem bestimmte ich das

1) M. Nussbaun, Zur Mechanik der Eiablage bei Rana fusea. Arch. f. mikr. Anat. Bd. 46. 1895 .

2) La Cellule. T. VII. 1891: Recherches sur l'appareil génital femelle de quelques Batraciens indigènes. 
Gewicht aller dieser Gebilde und konnte darans auch den Gewichtsanteil der Hülle am Gewicht des umbüllten Eies ermitteln.

Die Bauchhöhleneier uibertrug ich einzeln in Wagegläschen mittels länglicher dreieckiger Schnitzel von gehärtetem Fließpapier, mit deren Spitze ich sie auffing. Die Bauchhöhlenfltissigkeit wurde vorsichtig mit etwas Filtrierpapier von den in die Gläschen übertragenen Eier abgesangt, soweit sie nicht schon in das dieieckige Stiickchen übergegangen war.

Folgendes sind zunächst die Gewichte der Bauchhöhleneier, der mit Hülle versehenen Eileitereier und der leeren Hüllen. Es wurde stets das Gesamtgewicht einer größeren Menge Eier oder Hüllen durch deren Anzahl dividiert. Die Zahlen sind nicht als absolnt fur alle Froscheier guiltig zn betrachten, da die Eier, wie es durch entwicklungsmechanische Untersuchungen bekannt ist, in der Größe individuell etwas variieren.

Es wogen:

Das fertige Froschei (Banchhöhlenei) 0,001897 g, oder abgerundet annähernd $2 \mathrm{mg}$.

Das Ei mit Gallerthïlle $0,004674 \mathrm{~g}$, oder abgerundet 4 bis $5 \mathrm{mg}$.

Hieraus ergibt sich, daß die Gallerthülle in demjenigen Zustande der Quellung, wie man sie an den Eileitereiern vorfindet, $0,002777 \mathrm{~g}$ wiegt, somit mehr als die Hälfte des Gewichtes der Eier anf die wasserhaltige Hülle kommt.

Die leeren Eihtullen aus dem rechten Eileiter, welche von ungleicher Größe waren, wogen durchschnittlich je 0,00897 g.

Sie wogen somit dreimal soviel als die ein Ei umgebende Hülle.

Die Wasserbestimmungen ergaben, daß die fertigen Eier im Durchschnitt enthalten:

$$
\begin{aligned}
& \text { Wasser . . . . 52,5\%, } \\
& \text { Trockensubstanz . . 47,5\%. }
\end{aligned}
$$

Das Trocknen wurde ther Schwefelsäure im evakuierten Exsikkator vorgenommen.

Diese Zahlen sind das Mittel aus drei Bestimmungen, von denen zwei (2 und 3) annähernd übereinstimmen, während (1) um ein ganzes Prozent von dem kleineren Werte (2) differiert.

$\begin{array}{cccc} & 1 & 2 & 3 \\ \text { Wasser _. . . 51,7 } & 52,7 & 53,03, \\ \text { Trockensubstanz } & 48,3 & 47,3 & 46,97 .\end{array}$

Die Abweichungen sind wahrscheinlich teilweise auf Materialverschiedenheiten zurïckzuführen, die durch größere oder geringere 
Mengen noch anhaftender Bauchböhlenflüssigkeit bedingt sind, oder anderseits auf die nicht bei jeder Bestimmung in derselben Weise eintretende unvermeidliche Verdunstung. während des Sammelns der Eier in die Wagegläser.

Für die leeren Eihüllen erhielt ich (aus einer Bestimmung):

Wassergehalt . . 78,65\%,

Trockensubstan $z .21,35_{; 0}^{\circ}$.

Die mit Hülle versehenen Eileitereier enthielten:

$\begin{array}{ll}\text { Wasser . . . . } & 67,48 \% \\ \text { Trockensubstanz . } & .32,52 \%\end{array}$

Hieraus ergeben sich durch Berechnung folgende Zahlen:

Das Gewicht der trockenen leeren Hülle (rechter Eileiter) beträgt . . . . . . . . . . . . . . 0,00192 g, das Gewicht des trockenen fertigen Eies. . . . . . 0,000901 umbiillten Eileitereies. . 0,00152 -

Die Hülle eines Eileitereies allein beträgt somit trocken 0,000619Folglich beträgt auch die Trockensubstanz einer leeren Hülle etwa dreimal soviel als die Trockensubstanz der ein Ei umgebenden Hülle. Dieselbe Differenz besteht zwischen beiden Gebilden im feuchten Zustand.

Das für die weiteren Untersuchungen von mir gesammelte Material besteht aus getrockneten Eileitereiern nebst ihren Hüllen.

Es ist somit schließlich noch von praktischem Wert, zu wissen, wieviel davon auf das $\mathrm{Ei}$ selbst und wieviel auf die Hüllensubstanz zu rechnen ist.

Aus den obigen Zahlen ergibt sich, daß von diesem Material kommen:

$$
\begin{aligned}
& \text { auf Huillensubstanz } .40,72 \%, \\
& \text { - Eisubstanz } \quad . \quad 59,28 \% .
\end{aligned}
$$

Diese Zahlen sind verwendbar, jedoch ist ihre Genauigkeit in demselben Maße begrenzt, als die Hüllengröße variiert.

Den Wassergehalt des Froscheies hat H. KoLB ${ }^{1)}$ zu 57,40\% angegeben. Ich mußte diese Zahl als einzig vorhandene in eine Zusammenstellung der chemischen Zusammensetzung tierischer Eier aufnehmen. Sie beruht aber auf einem Irrtum KoLBs, wie ich so-

1) Heinz KolB, Chemische Untersuchung der Eier von Rana temporaria. Dissertation. Basel 1901. 
gleich näher auseinandersetzen werde, und muß durch die Zahl 52,5 ersetzt werden. Damit besitzt das Froschei den gleichen Wassergehalt wie das Sepia-Ei, für welches ich ihn zu 52,7\% ermittelt habe. Den nächst niedrigen weist das Gelbe des Hühnereies mit $51,00{ }_{0}$ auf, den nächst höheren das Ei von Maja squinado mit 56,4\%.

Die Zabl von KoLB ist deswegen irrtiumlich, weil sie sich auf das mit Gallerthülle versehene Eileiterei bezieht, jedoch ohne daß der Autor dies bemerkt hätte.

KoLB unterscheidet unentwickelten Laich, entwickelten ungelegten und gelegten befrucbteten Laich. Mit dem unentwickelten Laich sind die noch im Ovar befindlichen Eier oder einfach das Ovar gemeint, da dieses als Ganzes untersucht wurde. Entwickelten ungelegten Laich nennt KoLB die im Endabschnitt des Eileiters befindlichen Eier, welche schon mit der Gallerthiulle umgeben sind. Die dritte Art des Laichs und ihre Benennung bedarf keiner Erläuterung. KoLs findet für die Ovare einen Trockengehalt von $42,63 \%$ und für die umbüllten Eileitereier einen solchen von $30,24 \%$ und schließt daraus auf Wasserzunahme. Ich finde für die letzteren $32,5 \%$, eine Zahl, deren Abweichung von der KoLbschen wohl zum Teil auf Materialverschiedenheiten zurückzuführen ist sowie darauf, daß KoLı im Wärmeschrank bei erhöhter Temperatur getrocknet hat. Da die Eier selbst nach meinen Untersuchungen einen Trockengebalt von $47,5 \%$ haben und die Hüllen einen solchen von $21,35 \%$, so läßt sich darans der zwischen diesen beiden Zahlen liegende Trockengehalt der Eileitereier verstehen. Die von KoLb konstatierte Wasserzunahme des Eies kommt also nicht auf dessen Rechnung, sondern auf Rechnung der Umhtillung.

Das Übersehen der Hülle führte noch zu einer weiteren irrtiumlichen Schlußfolgerung. KoLB findet den Fettgehalt der Ovareier zu $19,1455 \%$, den der Eileitereier zu 11,918\%. Er schließt daraus, daß inzwischen eine Fettabnahme stattgefunden hat und nimmt als Ursache die Umwandlung des verschwundenen Fettes in Eiweiß an.

Der nur scheinbar geringere Fettgehalt des Eileitereies erklärt sich durch die Anwesenheit der Hülle, die natlirlich fettfrei ist.

Eine unnötige Genauigkeit ist die Berechnung der Prozente bis auf tausendstel und zehntausendstel Prozent in den obigen beiden Zahlen.

Besonders zu bemerken ist noch der Umstand, daß der Trockengehalt des Orars $(42,63 \%$ nach KoLs) anscheinend niedriger ist als der Trockengehalt der Banchhöhleneier $(47,5 \%$ nach meiner Fest- 
stellung). Das Ovar im ganzen ist somit wasserreicher als die fertigen Eier. Der größere Wasserreichtum läßt sich zum Teil daraus verstehen, daß die jüngeren Eier mit analysiert werden. Nach den Resultaten an den Ringelnattereiern verschiedener Größe ist anzunehmen, daß auch die jüngeren Froscheier einen höheren Wassergehalt besitzen werden. Ferner ist die um die Zeit der Eiablösung und Überwanderung in den Eileiter sowohl bei Schlangen wie auch bei Fröschen zu beobachtende starke Füllung der Bauchhöhle und Durchtränkung vieler Gewebe mit seröser Fltissigkeit zu beachten.

Die Übereinstimmung im Wassergehalt zwischen Sepia-Ei nnd Froschei zeigt zunächst, daß gleicher Wassergehalt und gleiche Größe bei Eiern verschiedener Arten nicht miteinander verbunden sind. Das Sepia-Ei zeigt $65,7 \mathrm{mg}$, das Froschei $2 \mathrm{mg}$. Ferner zeigt sich hierin, daß die Art der Farchung nicht im Zusammenhang mit dem Wassergehalt steht. Das Froschei zeigt totale Furchung, das Sepia-Ei Keimscheibenbildung. Was vom Wassergehalt gilt, gilt anch vom Fettgehalt. Das Froschei besitzt den höheren, das Sepia-Ei den niedrigeren Fettgehalt. Die Keimscheibenbildung kann also auch nicht vom höheren Fettreichtnm verursacht sein. Genauere Angaben über den Fettgehalt des Froscheies werden in einer späteren Mitteilung folgen.

Die beobachtete Störung der Überwanderung der Eileiter hatte, wie beschrieben, die Abscheidung einer großen Zahl leerer Eihüllen auf der rechten Seite zur Folge, welche sich im untersten Drittel des Eileiters ansammelten. Diese Anomalie ist geeignet, unsern Einblick in den Prozeß der Hüllenbildung des Froscheies zu erweitern.

Die Eier werden bekanntlich während des Durchtritts durch den Eileiter mit der Gallerthülle nmkleidet. Sie liegen dabei einzeln und die Hullen hängen nicht miteinander zusammen.

Die Gallerte besteht nach LeBRon ${ }^{1}$; aus zwei Schichten, einer inneren, mehr homogenen, dünnen, und einer äußeren, die etwa fünfmal so dick ist. Beide sind wieder konzentrisch geschichtet, die äußere gröber und unregelmäßiger, die innere sehr fein, dicht und regelmäßig ${ }^{2}$. Die innere Schicht stammt vom Anfangsteil des Eileiters $a b$, die iußere vom darauf folgenden Hauptabschnitt. Beide Ab-

1) L. c.

$\because$ Über weitere, nicht speziell strukturelle, interessante Eigenschaften der Gallerthitlle vgl. Roux, Ges. Abhandl. Bd. II. S. 100 und S. $16 \tilde{.}$ 
schnitte besitzen (ebenfalls nach LeBRuN) einen verschiedenen Bau. Das Anfangsteil (portion supérieure) besitzt als innerste Lage eine Schicht flimmernder Cylinderzellen. Es $\mathrm{mn} \beta$ angenommen werden, daß darunter auch secernierende Zellen sind. Allerdings finde ich dies bei Lebrux in demjenigen Abschnitt, in welchem er den Bau der Portion supérieure eingehend beschreibt, nicht besonders angegeben. Der mittlere (Haupt-) Abschnitt besitzt eine Schicht, die aus cylindrischen Drüsen gebildet ist, welche die Hauptmasse der Hiillsubstanz liefern.

Es ist nun merkwürdig, daß auch die Gallerte im rechten Uterns, in welchem sich, von ganz wenigen Eiern abgesehen, nur leere Hiillen befanden, die Form kleiner Kügelchen besitzt.

Vereinzelt findet man solche Kägelchen nicht selten bei der Entnahme von Eiern aus dem Eileiter. Diese Art des Vorkommens erwähnt auch Lebrun (S. 461). Er nennt die Gebilde Perlen und beschreibt sie als ganz durchscheinend. Tauche man sie jedoch in schwachen Alkohol, so erscheine in ihrem Centrum ein Knötchen, welches aus soliden Teilchen gebildet ist, am häufigsten aus abgeschuppten Epithelzellen. Ich kann dem nur noch hinzuftigen, daß die abweichende Beschaffenheit des Centrums anch ohne Anwendung von Alkohol sichtbar ist.

Charakteristischer als in solchen Einzelfällen tritt das Eigentiumliche dieser Erscheinung in dem hier mitgeteilten Vorkommen eines von leeren Eihüllen ganz angefüllten unteren Eileiterabschnittes hervor.

Wir sehen, daß die Gallerte auch ohne Anwesenheit von Eiern ebenso abgeschieden wird, als wenn Eier vorhanden wären. Die Abscheidung erfolgt zweckmäßig auf ein gedachtes Ziel hin. Nur der im Centrum befindliche Raum ist ersichtlich kleiner als wenn er durch ein Ei eingenommen wäre. - Wenn die Secretion durch die Anwesenheit eines Eies in Gang gesetzt würde und die Abscheidung in Form einer konzentrisch umschließenden Hille nur dureh das Ei bedingt wäre, so wäre die beobachtete Erscheinung unmöglich. Es ist somit keine Einrichtung vorhanden, welche den Eileiter nur bei Vorhandensein eines Eies secernieren ließe. Der konzentrische Bau, die Anordnung um einen Mittelpunkt und in Verbindung damit die Zerlegung in zahlreiche Perlen, Kügelchen oder Klümpchen erscheint somit als eine immanente Eigentumlichkeit des Secretes der Froscheileiter.

Die entsprechenden Secrete andrer nahe verwandter Tiere verhalten sich anders. Die Gallerte z. B., in welche die Eier der ge- 
meinen Kröte eingebettet sind, bildet einen kontinuierlichen Faden, in welchem in regelmäßigen Abständen die Eier liegen.

Zur weiteren Charakteristik der Gallerte der Froscheier führe ich noch folgendes aus Lebruns Arbeit an.

Öffnet man kurz nach dem Übertritt der Eier in den untersten Abschnitt des Eileiters diesen, so findet man die Eier in eine zähe, feste, anscheinend ungeteilte Masse vor. »Mais cette fusion n'est qu'apparente, elle paraît due à la présence d'une substance visqueuse déversée par les parties inférieures de l'oviducte, qui englue la masse des oufs, sans se fixer sur eux individuellement.

Lorsqu'on jette cette masse dans l'alcohol, elle perd son apparence homogène et on y découvre, que chaque ouf possède déjà une couche propre.*

Öffnet man den Eileiter erheblich später, so kann man sich auch ohne Anwendung von Alkobol von der Selbständigkeit der Hüllen der einzelnen Eier ibberzeugen, wie ibbrigens jedem bekannt ist, der sich mit der künstlichen Einzelbefruchtung von Froscheiern befaßt hat.

LEBRUN hat ebenfalls schon aus dem vereinzelten Vorkommen leerer Hüllen auf die Unabhängigkeit der Art der Hüllensubstanzausscheidung von der Anwesenheit der Eier geschlossen. Daher muf man auch nach ihm in bezug auf die Gallerte schließen, »que cette substance conserve quelque temps une énergie propre et une tendence à se grouper autour d'un centre. Cette tendence peut être aidée par l'élasticité de la paroi, qui doit exercer sur son contenu une action mécanique et par l'action des cils vibratils, qui tapissent la poche, font rouler les oufs et contribuent à distribuer à chacun sa part de substance fondamentales.

Die Aktion der Wimpern kann wohl für die Teilung in einzelne Kugeln nicht verantwortlich gemacht werden, da diese z. B. bei der Kröte trotz des Vorhandenseins von Wimpern nicht eintritt.

Übrigens ist aus dem Vorkommen einzelner leerer Gallertkugeln noch nicht sicher der Schluß auf Nichtbeteiligung der Eier an der Scheidung der einzelnen Gallertkugeln zu ziehen. Eine vereinzelte leere Hülle muß zwischen zwei Eiern mit Hülle gelegen haben und da ist es nicht auffällig, wenn eine zwischen ihnen gelegene Gallertmasse ein besonderes Klimpchen bildet. Konzentrischer Ban und Anordnung um ein Centrum läßt sich allerdings nicht einfach durch die Lage erklären.

Unser Fall schränkt aber gleichzeitig die Unabhängigkeit der Formierung der Gallertsubstanz von der Anwesenheit der Eier nach 
Einer Richtung hin ein. Während die Hüllen aller Eier ganz gleichmäßig groß sind, fanden sich unter den leeren Gallerthüllen solche von sehr angenfällig ungleicher Größe.

Ferner waren die Gallerthüllen im Durchschnitt bedeutend größer aìs die Gallerthülle eines einzelnen Eies.

Die feuchte Gallerthalle der Eier aus dem linken Eileiter wiegt $0,0028 \mathrm{~g}$, die feuchte leere Eihülle aus dem rechten Eileiter wiegt durchschnittlich $0,0090 \mathrm{~g}$ oder dreimal soviel.

Auf verschiedenem Grade der Quellung, also verschieden starker Wasseraufnahme, beruht die Differenz nicht, denn die trockene Hiblle eines Eileitereies betrug $0,0006 \mathrm{~g}$, die trockene leere Htille aus dem rechten Uterus betrug $0,0019 \mathrm{~g}$, also ebenfalls etwas mehr als dreimal soviel.

Somit ist das Ei zwar nicht die Ursache für die besondere Struktur der Hülle, wohl aber reguliert es ihre Größe. Sind keine Eier vorhanden, so finden wir die leeren Hüllen erstens ungleich, zweitens durchschnittlich von einer sehr viel bedeutenderen Größe als die der vollständigen Eier.

Gewinnen wir durch unsre Beobachtung auch einen Einblick in die Reize oder den Reiz, welcher die Secernierung des Secrets durch den Eileiter auslöst?

Wären im linken Eileiter $n u r$ leere Hüllen vorhanden, so würde dies unbedingt beweisen, daß die Gegenwart eines Eies im Eileiter nicht erforderlich ist, $\mathrm{um}$ ihn zur Secretion zu veranlassen. So aber finden wir einige wenige Eier vor und da läßt sich der Hergang in unserm Falle so denken:

Anfangs war der Eileiter zugänglich, einige Eier gelangten hinein und lösten den Secretionsvorgang ans. Darauf trat die Verlegung des Eileiters ein und es gelangten keine Eier mehr hinein. Dies hatte aber keinen Einfluß anf die Secretion; einmal eingeleitet, nahm sie ihren Fortgang. Dies soll zunächst nur eine Vorstellung sein, wie sie sich genau im Anschluß an unsre Beobachtnng bilden müßte.

Ganz sicher geht ans unserm Beispiel aber hervor, daß der einmal eingeleitete Vorgang der Abscheidang der Gallerte ohne Stillstand weiter abläuft. Welche besondere Ursache zur Einleitung dagegen erforderlich ist, läßt nnser Fall nicht ersehen.

LEBRTN führt folgende Beobachtung an, welche beweisen soll, daß die Secretion nicht direkt durch die Berührung des Eies mit der Wandung des Eileiters bewirkt wird. Man findet nämlich gewöhnlich einen dicken Strang von Gallertsubstanz weiter nach vorn als 
die ersten Eier liegen. Es ist daher weder die Erweiterung der Wandung, noch der Druck, den das Ei ausübt, dasjenige, was die Secretion veranlaBt.

Diese Beobachtung hindert uns aber meiner Meinung nach nicht, doch anzunehmen, daß der Eintritt des Eies die Einleitung zur Secretionsproduktion bildet und sie auslöst. Der Reiz bleibt eben nicht auf die Stelle der lokalen Berührung der Wand mit dem Ei beschränkt, sondern breitet sich sofort weiter auch auf die nach vorn auf dem zukünftigen Wege des Eies gelegene Strecke aus. Die Ausbreitung dürfte auf nervösem Wege geschehen.

LEBRUN nimmt dagegen drei Ursachen der Secretion an:

1) La maturité. A un moment donné la membrane céderait; la déhiscence serait naturelle et sans cause extrinsèque.

2) Les contractions de la presse abdominale.

3) Une compression produite par la turgeseence des vaisseaux sanguins. La congestion de l'oviducte: la couleur rosée qu'il prend alors suffit pour témoigner de l'afflux du sang qui s'y produit.

Von diesen drei Ursachen scheint mir die letzte beachtenswert. Die starke Füllung der Blutgefäße wird zweifellos im Zusammenhang mit der Secretion stehen. Eine physiologische Steigerung der Blutzufuhr ist zur Einleitung und Unterhaltung der Secretion gewiß erforderlich. Es kann aber darin nicht das auslösende primum movens gesucht werden. Die Frage würde darum so zu stellen sein, welche Ursache bewirkt die Füllung der Blutgefäße?

Die Kontraktion der Bauchpresse (2) scheint mir kein geeignetes Mittel zur Einleitung der Secretion. Sie müßte auf den Eileiter von allen Seiten einen Druck ausüben und somit würde auch gegen das dem Lumen zugekehrte Ende jeder Zelle die diesem gegenuiberliegende Seite der Röhre einen gleichen Gegendruck ausüben. Ferner besteht der erste Vorgang an jeder Zelle voraussichtlich in einer Veränderung der Beschaffenheit der Membran, welche eine jede Drüsenzelle und ihren Inhalt nach außen abschließt. Diese Veränderung kann nicht durch den Druck der Bauchpresse bewirkt werden. Die dritte Ursache LEBRcNs würde hier heranzuziehen sein.

Die zuerst genannte Ursache, nämlich der Zustand der Reife, ist nur die allgemeine Voraussetzung für Möglichkeit und Zustandekommen der Gallertabscheidung.

Somit möchte ich meine Ansicht dahin zusammenfassen, daß das erste eintretende Ei die einleitende Ursache zur Gallertabscheidung abgibt. 
Die Abscheidung ist nicht lokal an die Berührung der betreffenden Stelle mit dem Ei gebunden, sondern breitet sich (reflektorisch?) auf weiter entfernte Stellen aus. Es ist anch die Möglichkeit vorhanden, daß ein auf den einen Eileiter wirkender Reiz auch die Secretion im andern veranlaßt. Die Abscheidung geht nach Aufhören der einleitenden Ursache unverändert weiter.

$\mathrm{DaB}$ die besonderen Eigentümlichkeiten in der Struktur des Secrets von der Gegenwart des Eies unabhängig sind, wurde schon oben ausftihrlich dargelegt.

Die Größe der einzelnen secernierten Hüllenkörper wird dagegen vom $\mathrm{Ei}$ reguliert.

Es ist möglich, daß außer dem Ei anch andre in den Eileiter gelangende Körper den Secretionsvorgang veranlassen wtirden. Ein entsprechendes Experiment, welches wesentlich zur Aufklärung dienen wirde, konnte ich bisher noch nicht anstellen. 